\title{
Pressure induced valence and metal-insulator transitions in the Falicov-Kimball model with nonlocal hybridization
}

\author{
Pavol Farkašovský \\ Institute of Experimental Physics, Slovak Academy of Sciences \\ Watsonova 47, 04353 Košice, Slovakia
}

\begin{abstract}
We present a simple, but very realistic, model for a description of pressure induced valence and metal-insulator transitions in mixed valence systems. It is based on the extended Falicov-Kimball model and the supposition that the key interaction governing these transitions is the nonlocal hybridization between the localized $f$ and itinerant $d$ electrons. Taking into account, in addition, the parametrization between the external pressure and the $d$ - $f$ hybridization (the experimental fact), the model is able to describe, at least qualitatively, valence as well as metal-insulator transitions driven by the external pressure observed experimentally in some rare-earth systems, like $\mathrm{SmB}_{6}$.
\end{abstract}




\section{Introduction}

The valence and metal-insulator transitions belong certainly to the most popular manifestations of cooperative phenomena in solids. These transitions are observed in a wide group of substances formed by transition-metal oxides as well as rare-earth sulphides, halides and borides, when some external parameters (like pressure or temperature) are varied [1]. They are in many cases first-order phase transitions, however, second-order transitions ranging from very gradual to rather steep are also observed.

To describe all such transitions in a unified picture Falicov and Kimball [2] introduced a simple model in which only two relevant single-electron states are taken into account: extended Bloch waves and a set of localized states centered at the sites of the metallic ions in the crystal. It is assumed that valence and insulator-metal transitions result from a change in the occupation numbers of these electronic states, which remain themselves basically unchanged in their character. The only one quantity that can change in this model is the position of the $f$-level energy $E_{f}$. Then, considering a simple parametrization [3] between the external pressure $p$ and the position of $f$-level energy $\left(E_{f} \sim p\right)$ all valence changes as well as metal-insulator transitions induced by external pressure can be directly interpreted in terms of $E_{f}$.

The Hamiltonian of the Falicov-Kimball model can be written as the sum of three terms:

$$
H=\sum_{i j} t_{i j} d_{i}^{+} d_{j}+U \sum_{i} f_{i}^{+} f_{i} d_{i}^{+} d_{i}+E_{f} \sum_{i} f_{i}^{+} f_{i},
$$

where $f_{i}^{+}, f_{i}$ are the creation and annihilation operators for an electron in the localized state at lattice site $i$ with binding energy $E_{f}$ and $d_{i}^{+}, d_{i}$ are the creation and annihilation operators of the itinerant spinless electrons in the $d$-band Wannier state at site $i$.

The first term of (1) is the kinetic energy corresponding to quantum-mechanical hopping of the itinerant $d$ electrons between sites $i$ and $j$. These intersite hopping tran- 
sitions are described by the matrix elements $t_{i j}$, which are $-t$ if $i$ and $j$ are the nearest neighbours and zero otherwise. The second term represents the on-site Coulomb interaction between the $d$-band electrons with density $n_{d}=N_{d} / L=\frac{1}{L} \sum_{i} d_{i}^{+} d_{i}$ and the localized $f$ electrons with density $n_{f}=N_{f} / L=\frac{1}{L} \sum_{i} f_{i}^{+} f_{i}$, where $L$ is the number of lattice sites. The third term stands for the localized $f$ electrons whose sharp energy level is $E_{f}$.

It is interesting that this simple model is able to explain many physical aspects of real mixed valence systems, like the pressure induced continuous and discontinuous valence transitions as well as discontinuous metal-insulator transitions [4, 5] despite the fact that neglects a direct hybridization (between $d$ and $f$ orbitals) which is usually present in these systems. From this point of view it is important to ask what happens with the above mentioned picture of valence and metal-insulator transitions when a direct hybridization between the $d$ and $f$ orbitals is switched on. For the case of local $d$ - $f$ hybridization this question has been partially answered in our previous papers [5, 6]. Using the exact diagonalization and density-matrix-renormalization-group calculations we have found that the local hybridization smears the discontinuous character of valence transitions and stabilizes the insulating phase. However, in real mixed valence materials the local hybridization is forbidden (for parity reasons) and only the nonlocal one (with inversion symmetry) between the nearest-neighbour $f$ and $d$ orbitals, is allowed [7]. Thus for a correct description of valence and metal-insulator transitions in these materials one has to consider the nonlocal hybridization of the form $V_{i, j}=V\left(\delta_{j, i-1}-\delta_{j, i+1}\right)$ that leads to $k$-dependent hybridization of the opposite parity than corresponds to the $d$ band $\left(V_{k} \sim \sin (k)\right)$ [7]. This type of hybridization has been used, for example, in our very recent paper [8] to study the formation and condensation of excitonic bound states in the Falicov-Kimball model and it was shown that the system with the nonlocal hybridization exhibits fundamentally different behaviour than one with the local interaction. While the local hybridization strongly supports the for- 
mation of excitonc condensate, the nonlocal hybridization (with inversion symmetry) destroys it completely.

In the current paper we focus our attention on the problem of the pressure induced valence and metal-insulator transitions in mixed valence systems with nonlocal hybridization. However, unlike the previous studies, we use the fundamentally different approach. Indeed, while in the previous theoretical works [4,9] the pressure induced valence and metal-insulator transitions have been explained via the $E_{f}-p$ parametrization, in the current paper they are interpreted directly via the $V-p$ parametrization. For the case of nonlocal hybridization such a parametrization is physically very reasonable since the overlap (hybridization) of $d$ and $f$ orbitals located on the neighboring lattice sites increases with the increasing hydrostatic pressure, due to the decreasing lattice constant. We show that such a parametrization leads to a nice qualitative correspondence between the theoretical results obtained within the proposed approach and experimental measurements of the pressure dependence of valence and energy gap in $\mathrm{SmB}_{6}$, the compound at present very intensely studied as a potential candidate of a strongly correlated topological insulator [10].

\section{Results and Discussion}

In order to investigate possibilities for valence and insulator-metal transitions driven by the nonlocal hybridization (pressure) in the extended Falicov-Kimball model we have used the density-matrix-renormalization-group (DMRG) method [1] that allows to treat relatively large clusters $(L \sim 100)$ and still to keep the high accuracy of computations. We typically keep up to 128 states per block, although in the numerically more difficult cases, where the DMRG results converge slower, we keep up to 500 states. Truncation errors, given by the sum of the density matrix eigenvalues of the discarded states, vary from $10^{-8}$ in the worse cases to zero in the best cases.

Let us first discuss the picture of metal-insulator transitions obtained within the 
Falicov-Kimball model with the nonlocal hybridization. The nature of the ground state has been identified through the behavior of the single particle excitation energy defined as $\Delta(N)=E_{G}(N+1)+E_{G}(N-1)-2 E_{G}(N)$, where $E_{G}(N)$ is the ground state energy for $N$ electrons (here we consider the half-filed band case when the number of electrons $N$ equals to the number of lattice sites $L$ ). Then by a definition [12], the metallic state corresponds to $\Delta(L=\infty)=0$ and the insulating one to $\Delta(L=\infty)>0$. The results of our numerical calculations for $\Delta$ as a function of nonlocal hybridization (pressure) are summarized in Fig. 1 for several different values of the f-level position $E_{f}$ and several different cluster sizes $L$. One can see that finite size effects are still present,
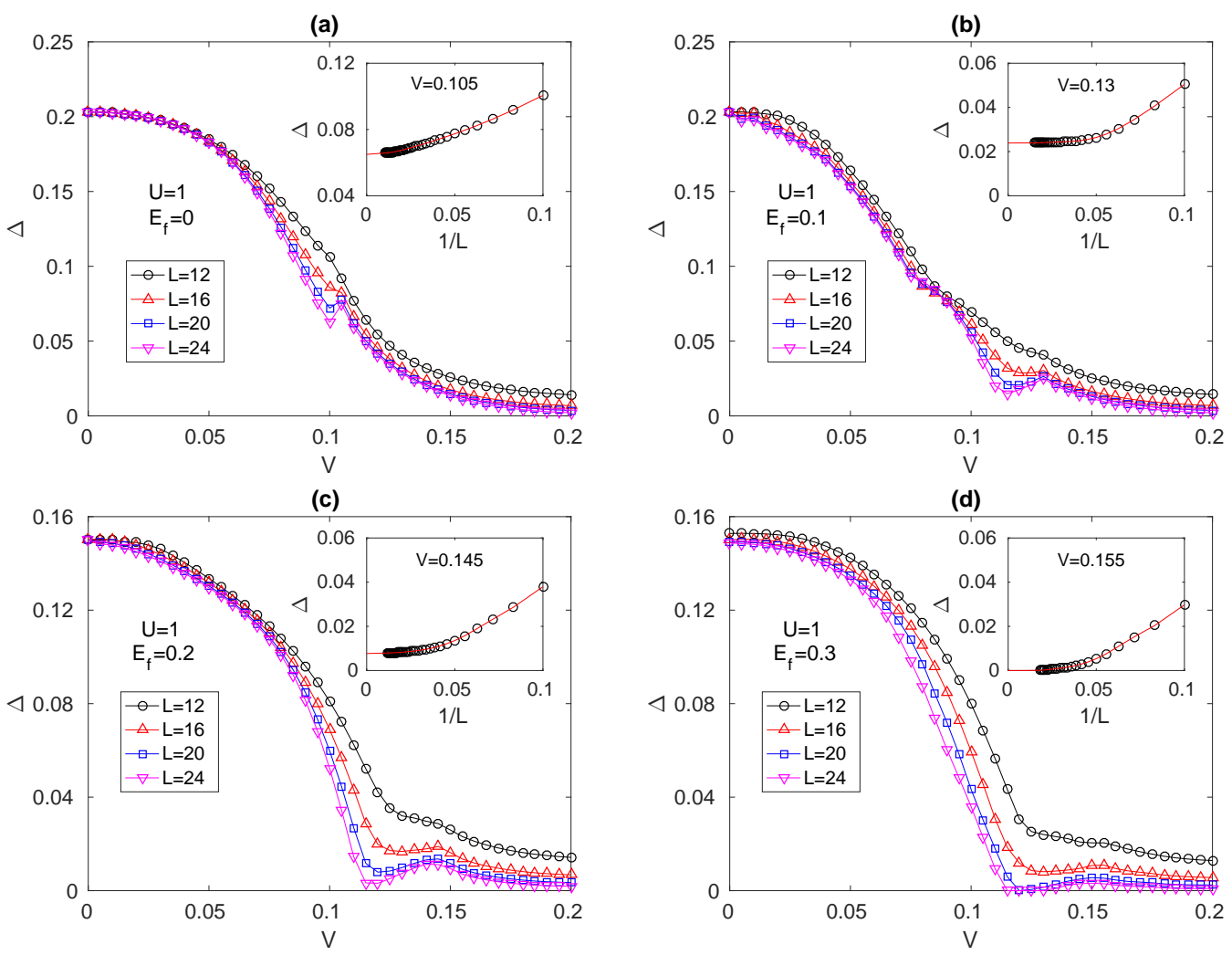

Figure 1: The single particle excitation energy $\Delta$ as a function of the nonlocal hybridization $V$ calculated for several different values of the $f$-level energy $E_{f}$ on several different clusters at $U=1$. The insets show the $1 / L$ dependence of $\Delta$ at the position of the local maximum.

but despite this fact the general trends in behaviour of the single particle excitation energy $\Delta$ as a function of nonlocal hybridization $V$ are obvious. The most obvious of them is the existence of a critical hybridization $V_{c} \sim 0.1$, below and above which 
the single particle excitation energy behaves fully differently. Indeed, for $V<V_{c}$ the single particle excitation energy is gradually reduced with increasing $V$, but at $V=V_{c}$ it starts to increase again, reaches its local maximum at $V_{0}$ and then continuously vanishes. The insulating character of the ground state below $V_{c}$ is obvious, however the same can not be said for $V>V_{c}$, where relatively strong finite-size effects are observed. To reveal the nature of the ground state in this region, we have performed a detailed finite-size scaling analysis of the $L$ dependence of $\Delta$ at the point of local maximum $V_{0}$. The resultant behaviors of $\Delta$ as a function of $1 / L$ are shown in the insets to Fig. 1 and they clearly demonstrate that the ground state of the model at $V=V_{0}$ is insulating for $E_{f}=0,0.1,0.2$ and metallic for $E_{f}=0.3$. This indicates that there exists some critical value of the $f$-level energy $E_{f}=E_{f}^{c} \sim 0.3$, above which the system exhibits by the nonlocal hybridization induced the insulator-metal transition at the critical point $V=V_{c}$. To verify this conjecture we have performed (for $E_{f}=0.3$ ) the exhaustive finite-size scaling analysis of the $L$ dependence of $\Delta$ for a wide set of $V$ values from the region $V<V_{c}$ as well as $V>V_{c}$. The results of numerical calculations are displayed in Fig. 2a and they fully confirm our conjecture, at $V=V_{c}$ the system undergoes the insulator-metal transition induced by nonlocal hybridization $V$. A direct
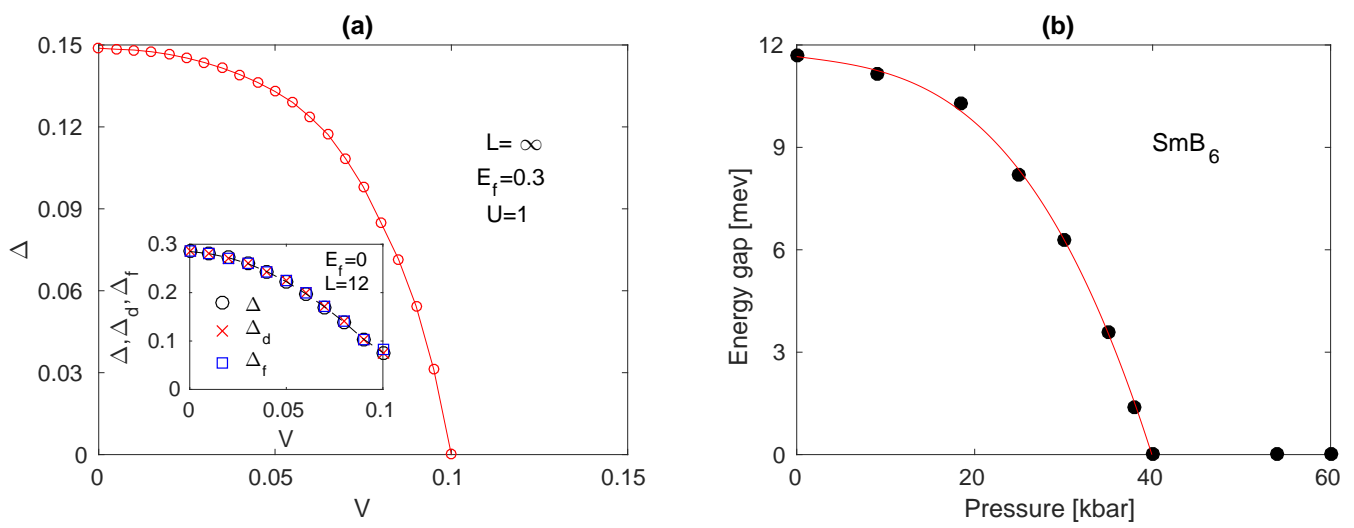

Figure 2: (b) The extrapolated single particle excitation energy $\Delta$ as a function of the nonlocal hybridization $V$ calculated for $E_{f}=0.3$ and $U=1$. The inset show $\Delta, \Delta_{f}$ and $\Delta_{d}$ as function of $V$ calculated at $E_{f}=0, U=1$ and $L=12$. (b) Pressure dependence of the energy gap in $\mathrm{SmB}_{6}[13$. 
comparison of our numerical results, obtained for the single particle excitation energy $\Delta$ as a function of the nonlocal hybridization (see Fig.2a), with the experimental ones obtained for the energy gap as a function of pressure in the $\mathrm{SmB}_{6}$ compound [13] (see Fig.2b), shows that there is a nice correspondence between the theoretical and experimental results when one takes into account the $V-p$ parametrization instead of the $E_{f}-p$ parametrization. Of course, this comparison will be correct only if we show that the single particle excitation energy is proportional to the energy gap at the Fermi level $E_{F}$. For this reason we have calculated directly the single particle excitation energy $\Delta$ and the $f$ and $d$ electron density of states and the corresponding $f$ and $d$ electron gap $\left(\Delta_{f}\right.$ and $\left.\Delta_{d}\right)$ at the Fermi level $E_{F}$ on the small finite cluster of $L=12$ sites. The results of numerical calculations obtained for several different values of nonlocal hybridization are shown in the inset to Fig. 2a and they clearly demonstrate that $\Delta, \Delta_{f}, \Delta_{d}$ coincide for all examined values of nonlocal hybridization, what validates the above discussed correspondence.

Let us now turn our attention to the problem of valence transitions in the FalicovKimball model with the nonlocal hybridization in the context of $V-p$ parametrization. Our DMRG results for the $f$-electron occupation number $n_{f}$ obtained for several different values of $f$-level position $E_{f}$ are shown in Fig. 3a. Comparing these results with ones obtained in our previous paper within the $E_{f}-p$ parametrization [9] one can find the significant difference. Indeed, while in the case of the $E_{f}-p$ parametrization only very small effects of nonlocal hybridization on the behavior of the $f$-electron occupation number $n_{f}$ (valence transitions) have been observed (especially for $V<V_{c}$ ), the $V-p$ parametrization is able to produces large valence changes over the relatively narrow interval of $V(\mathrm{p})$ values. From the point of view of above discussed correspondence between the theoretical results obtained for the single particle excitation energy within the $V-p$ parametrization and experimental results obtained for the pressure dependence of the energy gap in $\mathrm{SmB}_{6}$, it is interesting to make the same 

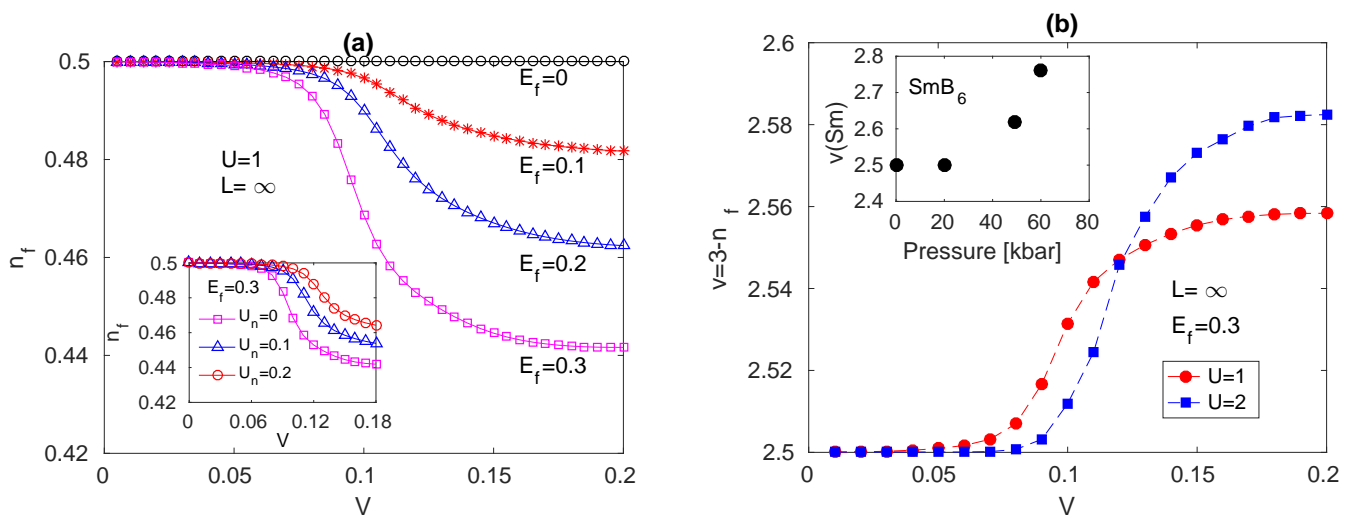

Figure 3: (a) The $f$-electron occupation $n_{f}$ as a function of nonlocal hybridization $V$ calculated for several different values of the $f$-level energy $E_{f}$ at $U=1$ and $L=\infty$. The inset shows the $f$-electron occupation $n_{f}$ as a function of $V$ calculated for several different values of the nonlocal Coulomb interaction $U_{n}$ between the $f$ and $d$ electrons at $E_{f}=0.3, U=1$ and $L=\infty$. (b) The valence $v=3-n_{f}$ as a function of nonlocal hybridization calculated for two different values of the Coulomb interaction $U$ at $E_{f}=0.3$ and $L=\infty$. The inset shows the pressure dependence of samarium valence in $\mathrm{SmB}_{6}$ [14].

comparison also for the $f$-electron occupation number $n_{f}$. Since the valence $v$ of the rare-earth ion in the mixed valence systems is directly connected with the $f$-electron occupation number $n_{f}$ (e.g., $v=3-n_{f}$ for $\mathrm{SmB}_{6}$ ) such a comparison is possible thanks experimental data presented in [14] for the pressure dependence of samarium valence in $\mathrm{SmB}_{6}$ compound. Both the theoretical and experimental results for the averaged valence are compared in Fig. 3b and again one can find a good correspondence between the theoretical and experimental results when the $V-p$ parametrization is considered. To reveal the effects of Coulomb interaction on the valence transitions we have plotted in Fig. 3b the hybridization dependence of the average valence $v=3-n_{f}$ for two different values of the Coulomb interaction $U=1$ and $U=2$. The results obtained show that the correlation effects (the Coulomb interaction) enhance considerably the average valence of rare-earth ions in mixed valence systems.

Since our model considers only the local Coulomb interaction, it is natural to ask, if the above described picture of valence transitions persists also in the physically more realistic case, when the nonlocal Coulomb interaction between the $d$ and $f$ orbitals is switched on. To answer this question we have extended our model Hamiltonian (1) by 
the nonlocal nearest-neighbour $d$ - $f$ Coulomb interaction $H_{n}=U_{n} \sum_{i, j} d_{i}^{+} d_{i} f_{j}^{+} f_{j}$ and calculated the $f$-electron occupation number $n_{f}$ as a function of $V$ for several different values of $U_{n}$. The results of our numerical calculations are presented in the inset to Fig. 3a and they clearly demonstrate that results obtained for $U_{n}=0$ persist also for finite and physically reasonable values of $U_{n}\left(U_{n} \sim V\right)$.

In summary, the DMRG method is used to examine effects of nonlocal hybridization on valence and metal-insulator transitions in mixed valence systems. On the basis of our numerical results we have proposed a simple, but very realistic, model for a description of pressure induced valence and metal-insulator transitions in these systems. Instead of the standard $E_{f}-p$ parametrization we have considered the physically more realistic $V-p$ parametrization. Under this assumption the model is able to describe, at least qualitatively, the valence as well as metal-insulator transitions driven by the external pressure observed experimentally in some rare-earth systems, like $\mathrm{SmB}_{6}$.

This work was supported by the Slovak Grant Agency VEGA under Grant 2/0112/18. Calculations were performed in the Computing Centre of the Slovak Academy of Sciences using the supercomputing infrastructure acquired in project ITMS 26230120002 and 26210120002 (Slovak infrastructure for high-performance computing) supported by the Research and Development Operational Programme funded by the ERDF. 


\section{References}

[1] D. L. Khomskii, Quantum Theory of Solids, edited by I.M. Lifshitz (Mir, Moscow $1982)$.

[2] L. M. Falicov and J. C. Kimball, Phys. Rev. Lett. 22, 997 (1969).

[3] C.E.T. Goncalves da Silva and L.M.Falicov, Solid State Commun. 17, 1521 $(1975)$.

[4] P. Farkašovský, Phys. Rev. B 51, 1507 (1995); 52, R5463 (1995).

[5] P. Farkašovský, Acta Physica Slovaca 60, 497 (2010).

[6] P. Farkašovský, Z. Phys. B 104, 553 (1997).

[7] G. Czycholl, Phys. Rep. B 143, 277 (1986).

[8] P. Farkašovský, Phys. Rev. B 95, 045101 (2017).

[9] P. Farkašovský, Phys. Rev. B 70, 035117 (2004).

[10] D. Chowdhury, I. Sodemann, and T. Senthil, Nature Communications 9, 1766 (2018).

[11] S. R. White, Phys. Rev. Lett. 69, 2863 (1992).

[12] T. Kennedy and E.H. Lieb, Physica A 138, 320 (1986).

[13] S. Gabani, E. Bauer, S. Berger, Y. Paderno, C. Paul, V. Pavlik, and N. Shitsevalova, Phys. Rev. B 67, 172406 (2004).

[14] K. Nishiyama, T. Mito, G. Pristas, Y. Hara, T. Koyama, K. Ueda, T. Kohara, Y. Akahama, S. Gabani, M. Reiffers, K. Flachbart, H. Fukazawa, Y. Kohori, N. Takeshita, and N. Shitsevalova JPS Conf. Proc.. B 3, 011085 (2014). 